fibrosis of different organs. The occurrence of endothelial dysfunction together with fibrosis indicates that endothelial cell-derived factors, such as endothelin-1 (ET-1), may have an important role in the pathogenesis of SSc. The upregulation of ET-1 activates inflammatory cells and leads to nitric oxide synthase inhibition associated with arterial stiffness.

Objectives: The purpose of this study was to evaluate ET-1 serum levels in women with systemic sclerosis (SSc) compared with healthy controls and to examine possible associations between ET-1 and markers of arterial stiffness. Methods: This cross-sectional study was performed in San Cecilio Hospital, Granada (Spain) from November 2017 to May 2019. Sixty-two women with SSc and 62 age and sex matched healthy controls were enrolled in this study. Pulse Wave Velocity (PWV) was measured non-invasively along the carotid-femoral arterial segment. Serum ET-1 was analysed using indirect enzyme-linked immunosorbent assay (ELISA).

Results: A total of 62 female patients were included in our study, with a mean (SD) age of $53 \pm 10$ years. The majority were Caucasian $(90.5 \%)$. The mean disease duration was $8.8 \pm 6.9$ years. Forty-four $(70.9 \%)$ patients had a limited form of the disease and $18(29.1 \%)$ had a diffuse form

There was a significant difference in ET-1 serum levels between SSc female patients and healthy controls ( $28.4 \pm 10.6$ vs. $21.1 \pm 11.7 \mathrm{pg} / \mathrm{ml}, \mathrm{p}=0.001)$. Serum levels of ET-1 were positively associated with PWV $(r=0.26, p<0.05)$, within the study group. In addition, in the linear regression model, higher ET-1 concentrations were associated with higher PWV $[\beta=0.0395 \% \mathrm{Cl}(0.001,0.060) ; \mathrm{p}<$ 0.05].

Conclusion: This study shows that ET-1 serum levels are associated with PWV in women with SSc. Therefore, drugs that block ET-1 may be effective in reducing large artery stiffness in women with SSc, and thus cardiovascular risk. REFERENCES:

[1] LeRoy EC, Black C, Fleischmajer R, Jablonska S, Krieg T, Medsger TA Jr, et al. Scleroderma (systemic sclerosis): classification, bubsets and pathogenesis. J Rheumatol 1988; 15(2):202-205.

[2] Shi-Wen X, Denton CP, Dashwood MR, Holmes AM, Bou-Gharios G, Pearson JD, et al. Fibroblast matrix gene expression and connective tissue remodeling: role of endothelin-1. J Invest Dermatol 2001; 116(3):417-425.

[3] Heintz B, Dörr R, Gillessen T, Walkenhorst F, Krebs W, Hanrath P, et al. Do arterial endothelin 1 levels affect local arterial stiffness?. Am Heart J 1993; 126 (4): 987-989.

[4] McEniery CM, Qasem A, Schmitt M, Avolio AP, Cockcroft JR, Wilkinson IB. Endothelin-1 regulates arterial pulse wave velocity in vivo. J Am Coll Cardiol 2003; 42 (11): 1975-1981.

Disclosure of Interests: None declared

DOI: 10.1136/annrheumdis-2021-eular.304

\section{AB0407 CARDIOVASCULAR BURDEN IN SYSTEMIC SCLEROSIS: QRISK3 VERSUS FRAMINGHAM FOR RISK ESTIMATION}

M. Di Battista ${ }^{1}$, S. Barsotti ${ }^{1}$, A. Della Rossa ${ }^{1}$, M. Mosca ${ }^{1}{ }^{1}$ University of Pisa, Rheumatology Unit, Pisa, Italy

Background: Cardiovascular (CV) diseases, namely myocardial infarction and stroke, are not among the most known and frequent complications of systemic sclerosis (SSc), but there is growing evidence that SSc patients have a higher prevalence of $\mathrm{CV}$ diseases than the general population [1].

Objectives: To compare two algorithms for CV risk estimation in a cohort of patients with SSc, finding any correlation with clinical characteristics of the disease. Methods: SSc patients without previous myocardial infarction or stroke were enrolled. Traditional CV risk factors, SSc-specific characteristics and ongoing therapies were assessed. Framingham and QRISK3 algorithms were then used to estimate the risk of develop a CV disease over the next 10 years.

Results: Fifty-six SSc patients were enrolled. Framingham reported a median risk score of $9.6 \%$ (IQR 8.5$)$, classifying 24 (42.9\%) subjects at high risk, with a two-fold increase of the mean relative risk in comparison to general population. QRISK3 showed a median risk score of $15.8 \%$ (IQR 19.4), with $36(64.3 \%)$ patients considered at high-risk. Both algorithms revealed a significant role of some traditional risk factors and a noteworthy potential protective role of endothelin receptor antagonists $(p=0.003)$. QRISK3 was also significantly influenced by some SSc-specific characteristics, as limited cutaneous subset $(p=0.01)$, interstitial lung disease $(p=0.04)$ and non-ischemic heart involvement $(p=0.03)$, with the first two that maintain statistically significance in the multivariate analysis ( $\mathrm{p}=0.02$ for both).

Conclusion: QRISK3 classifies more SSc patients at high-risk to develop CV diseases than Framingham, and it seems to be influenced by some SSc-specific characteristics. If its predictive accuracy were prospectively verified, the use of QRISK3 as a tool in the early detection of SSc patients at high CV risk should be recommended.

REFERENCES:

[1] Ngian GS, Sahhar J, Proudman SM, Stevens W, Wicks IP, Van Doornum S. Prevalence of coronary heart disease and cardiovascular risk factors in a national cross-sectional cohort study of systemic sclerosis. Ann Rheum Dis. 2012;71:1980-3.

Disclosure of Interests: None declared

DOI: 10.1136/annrheumdis-2021-eular.327

\section{AB0408 \\ SYSTEMIC SCLERODERMA AND ENVIRONMENTAL RISK FACTORS: IDENTIFYING ASSOCIATIONS MINING THE BIOMEDICAL LITERATURE}

A. Rodriguez-Pla ${ }^{1}$. 'Mayo Clinic Scottsdale Campus, Rheumatology,

Scottsdale, United States of America

Background: A debate still exists concerning the role of occupational and environmental factors in the pathogenesis of systemic scleroderma (SSc).

Objectives: Our aim was to explore associations between SSc and environmental factors utilizing an automatic semantic interpretation of PubMed results. Methods: The literature search string: ("systemic sclerosis" OR "scleroderma") AND ("occupational exposure" OR "environmental" OR "risk factor") was used to retrieve abstracts from the entire PubMed database, using Semantic MEDLINE 2 , on $6 / 14 / 2020$. This application represents a network of semantic predications (triples of the form subject-predicate (or relation) -object, e.g. Occupational Exposure causes Systemic Scleroderma) on a knowledge graph. Subject and object arguments of each predication are concepts from the Unified Medical Language System (UMLS) Metathesaurus and the relation is taken from the UMLS Semantic Network. The system allows for choosing the central topic ("Systemic Scleroderma"), the length of the network (3 nodes), and automatic summarization, eliminating the less informative predications.

Results: The search string retrieved 864 citations and identified 6,397 predications by using 34 types of relations. Initially, we focused our attention on the 'CAUSES' type of relation (Figure 1), displaying a network with 59 nodes and 57 edges.

The central concepts of this network, identified as having causal relationship with SSc are autoimmune diseases/autoimmunity, chemicals such as bleomycin occupational and environmental exposure, especially silica, vinyl chloride and trichloroethylene, genes, including HLA and non-HLA genes, genetic polymorphisms, transcription factors (TFs) such as Fli1 and KLF5, and fibrosis. Eosinophilia-myalgia syndrome, toxic oil syndrome and infection were all causally linked to autoimmune diseases. Minerals were associated with occupational exposure and with autoimmune diseases. Concepts causally linked to fibrosis were rare diseases, HLA genes, other non-HLA genes, such as STAT4, IR4, IR5, TLR4 TLR7 and Rho-associated Kinase, and vinyl chloride monomer. Pathogenic factors associated with SSc were endothelial dysfunction and extracellular matrix proteins. Many of the papers in the network also suggested that hormonal factors are involved.

Conclusion: Inspection on the knowledge graphs reveals concepts central to research on the etiopathogenesis of SSc. The relations in which these concepts participate, provide more specific information. The Semantic MEDLINE graph supports the kind of patterns that underpin literature-based discovery.

Although the pathogenesis of SSc remains elusive, it is accepted that initial vascular damage driven by autoimmunity and environmental factors causes abnormalities in the vasculature resulting in the activation of fibroblasts in various organs. Silica and solvents such as trichloroethylene seem to be the most consistently suspected environmental agents in SSc.

\section{REFERENCES:}

[1] Rindflesch TC,et al. Semantic MEDLINE: An advanced information management application for biomedicine. Information Services \& Use 2011;31:15-21.

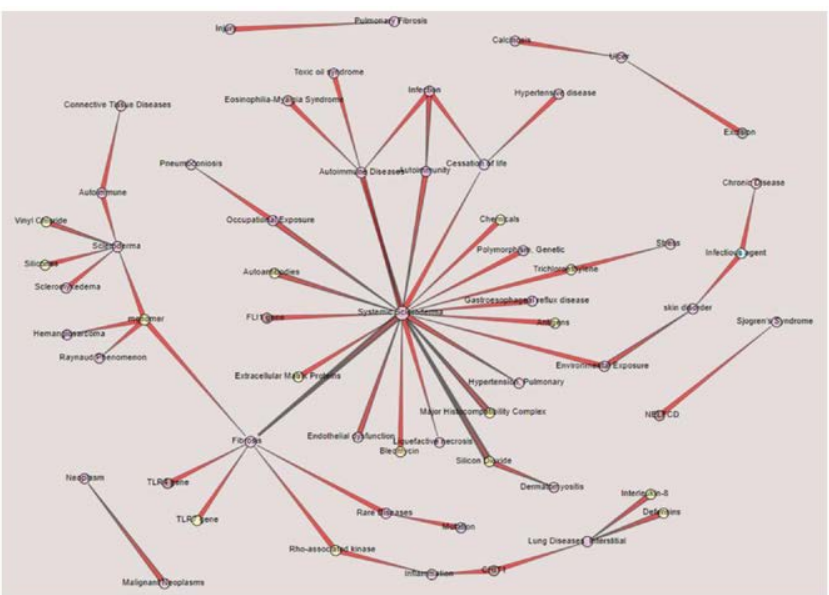

Figure 1. Semantic Network of Casual Relationships of Systemic Scleroderma. 